\title{
Comparative study of two contrast agents for intraoperative identification of sentinel lymph nodes in patients with early breast cancer
}

\author{
Yan Sun ${ }^{1}$, Ligang Cui ${ }^{1}$, Shunmin Wang ${ }^{1}$, Tan Shi ${ }^{1}$, Yunxia Hao ${ }^{1}$, Yutao Lei $^{2}$ \\ ${ }^{1}$ Department of Ultrasound, Peking University Third Hospital, Beijing, China; ${ }^{2}$ Department of General Surgery, Peking University Third Hospital, \\ Beijing, China \\ Contributions: (I) Conception and design: Y Sun, Y Lei; (II) Administrative support: L Cui, S Wang; (III) Provision of study materials or patients: \\ Y Lei; (IV) Collection and assembly of data: Y Sun, T Shi; (V) Data analysis and interpretation: Y Hao; (VI) Manuscript writing: All authors; (VII) \\ Final approval of manuscript: All authors. \\ Correspondence to: Yutao Lei. Department of General Surgery, Peking University Third Hospital,49 North Garden Road, Haidian District, Beijing \\ 100191, China. Email:sunyanka@sina.com.
}

\begin{abstract}
Background: The use of contrast-enhanced ultrasound (CEUS) to locate sentinel lymph nodes (SLNs) in breast cancer has been studied more and more in recent years. This prospective study aimed to compare periareolar injection of two different contrast agents, SonoVue ${ }^{\circledR}$ (SNV) and Sonazoid ${ }^{\circledR}$ (SNZ), followed by CEUS to identify SLNs in breast cancer patients with clinically negative nodes.

Methods: A total of 205 patients with T1-2N0M0 breast cancer were divided into the SNV group and SNZ group. All were administered a periareolar injection of SNV or SNZ and underwent US to identify contrast-enhanced SLNs. Each contrast-enhanced SLN underwent a biopsy with blue dye and examined again by CEUS in vitro.

Results: In all cases, contrast-enhanced lymphatic vessels were clearly visualized using US soon after the periareolar injection of SNZ, and the SLNs were easily identified. The SLN identification rates were 75.27\% (210/279) for SNV and 93.58\% (102/109) for SNZ. Although the accuracy of detecting SLN metastasis was slightly different between the two groups, there was no statistically significant difference between those groups $(\mathrm{P}=0.615)$. Moreover, it was possible to identify SLNs in vitro in the SNZ group, and these could be compared with the lymph nodes (LNs) located using SNZ during the preoperative stage and with blue dye during the procedure. This helped in determining the resection requirements.

Conclusions: When comparing the subdermal use of SNV and SNZ, no significant differences in the number of detected SLNs and the diagnosis of metastatic LNs were observed. Because SLNs can be detected for a longer time in living tissues with SNZ, this contrast agent may provide more intraoperative information for complete resection of all preoperative localization of SLN.
\end{abstract}

Keywords: Sentinel lymph node (SLN); contrast media; ultrasonography; breast neoplasms; biopsy

Submitted Feb 09, 2021. Accepted for publication Mar 18, 2021.

doi: $10.21037 / g s-21-87$

View this article at: http://dx.doi.org/10.21037/gs-21-87

\section{Introduction}

For patients with breast cancer, the axillary lymph node (ALN) status is an important prognostic factor that provides valuable information for decisions regarding adjuvant therapy. A sentinel lymph node (SLN) is the first node in the lymphatic chain draining a primary tumor. According to clinical standards, lymph node metastasis is determined by lymph node biopsy and lymphadenectomy (1). SLN biopsy (SLNB) is an invasive surgical procedure, which consists of two parts: lymphatic localization and surgical 
resection. In order to map sentinel lymph nodes, doctors performed preoperative lymphoscintigraphy to identify areas of increased extratumoral radiotracer accumulation (2). Then, surgeons inject blue dye near the site of the tumor to detect SLNs. Subsequently, surgical resection of the SLNs is performed, and the resected specimens are used to assess the presence of metastatic disease in the LNs.

CEUS has become an established screening modality used for various clinical indications of SLNs (3). SonoVue ${ }^{\circledR}$ (SNV) (Bracco Imaging SpA, Maderno, Italy), a blood pool agent, and Sonazoid ${ }^{\circledR}$ (SNZ) (GE Healthcare, Oslo, Norway), which exhibits an additional Kupffer phase, are contrast agents approved for liver imaging (4). Recent studies have shown that SNV has great potential for locating SLNs and diagnosing breast cancer SLN metastasis. However, SNZ has good stability and may have greater potential in superficial US. To our knowledge, no studies have compared the efficacy of SNZ and SNV in superficial US because few countries are currently using both contrast agents. Although a few studies have reported the efficacy of SNZ-enhanced US compared to that of SNV for the diagnosis of focal liver lesions (5), available results of the use of these two contrast agents during clinical studies of SLNs in patients with breast cancer are lacking. Therefore, this clinical study compared the ability of these two US contrast agents to detect and characterize SLNs in patients with early breast cancer. We present the following article in accordance with the STARD reporting checklist (available at http://dx.doi.org/10.21037/gs-21-87).

\section{Methods}

The study was conducted in accordance with the Declaration of Helsinki (as revised in 2013). This study was approved by the ethics committee of medical science research at Peking University Third Hospital (item number M2017394), and informed consent was obtained from all patients. Patients with T1-2N0M0 breast cancer were enrolled in this prospective study at Peking University Third Hospital between January 2018 and May 2020. Conventional US and careful palpation were performed to determine the ALN status of each patient. When ALN metastasis was suspected, fine-needle aspiration cytology was performed and such LNs were excluded from the study. Patients with a history of breast or axillary surgery and those who had undergone radiotherapy or chemotherapy were excluded from the study, Patients allergic to eggs, pregnant, or lactating were also excluded.

Finally, 205 patients were enrolled in this study (204 female and 1 male). The mean patient age was $53.54 \pm 12.32$ years (range, $19-75$ years). All patients underwent routine US and percutaneous CEUS for the evaluation of SLNs before the SLNB procedure.

US examinations were performed using GE LOGIQ ${ }^{\mathrm{TM}}$ E9 (GE Healthcare, Inc., Chicago, IL, USA) equipped with high-frequency linear array probes with a frequency of $9 \mathrm{MHz}$ and contrast pulse sequence imaging technology. Microbubble contrast agents (SNV and SNZ) were used with US during this study. Taking March 30, 2019 as the time node, SNV was used for all patients before this date, and SNZ was used for all patients starting in April 2019. Patients were placed in the supine position and examined using US and color Doppler. US contrast agent was injected around the areola at the 12:00, 3:00, 6:00, and 9:00 positions under general anesthesia. The total SNV and SNZ injections were $2 \mathrm{~mL}$ and $0.4 \mathrm{~mL}$, respectively.

Patients were divided into the SNV group and SNZ group according to the use of the different US contrast agents. The number, location, and mode of enhancement of LNs were observed and recorded in real time, along with the surface location. SLNs were marked on the skin surface, and the number, shape, size, and location (the depth from the skin and the distance from the lateral edge of the pectoralis major muscle) of the SLNs were recorded. After contrast-enhanced US, $2 \mathrm{~mL}$ of $1 \%$ blue dye (Methylene blue) was injected subcutaneously around the areola at the 12:00, 3:00, 6:00 and 9:00 positions. During the dissection procedure, lymphatic vessels and LNs were separated according to the markings of the blue dye tracing. The lymphatic vessels and SLNs were observed, compared with the SLN markings resulting from the preoperative CEUS localization method, and recorded. The SLNs of the different contrast groups and the corresponding bluedye markings were compared after surgery. Furthermore, all excised SLNs and axillary samples, soaked in normal isotonic physiologic saline, were reevaluated in vitro using CEUS.

\section{Statistical analysis}

During this study, SPSS 22.0 software (SPSS Inc., Chicago, IL, USA) was used for data analysis. The quantitative data are expressed as mean \pm standard deviation (SD), and the numbers of SLNs detected using percutaneous CEUS and 
SLNB were compared using the t-test and Fisher's exact test. The accuracy of percutaneous CEUS and the number and localization of SLNs were calculated and compared with the surgical results. $\mathrm{P}<0.05$ was considered statistically significant.

The diagnosis of SLN metastasis was independently determined by two experienced physicians with at least 3 years of CEUS experience and who were blinded to the medical history and test results of the patients. If both physicians declared the same evaluation results, a CEUSdiagnosed SLN metastasis was concluded. If there were discrepancies in their results, then the cine clips were assessed by a third physician with CEUS experience. The pathological results were used as the reference standard to calculate the sensitivity, specificity, accuracy, positive predictive value (PPV), and negative predictive value (NPV)

Table 1 Patient characteristics

\begin{tabular}{lcc}
\hline & SNV Group & SNZ Group \\
\hline Cases, $\mathrm{n}$ & 135 & 68 \\
Age, years & $53.34 \pm 12.44$ & $53.79 \pm 12.21$ \\
Tumor histology & & 6 \\
DICS & 21 & 1 \\
Paget & 2 & 53 \\
NSIC & 92 & 8 \\
Others & 20 & 102 \\
SLN & & 17 \\
No. of SLNs & 210 & 26 \\
Metastatic cases & 29 & \\
Metastatic SLNs & 38 & \\
\hline
\end{tabular}

SNV: SonoVue ${ }^{\circledR} ;$ SNZ: Sonazoid ${ }^{\circledR} ;$ SLN, sentinel lymph node; DICS, ductal carcinoma in situ; NSIC, non-specific invasive cancer. of CEUS for the diagnosis of SLN metastasis.

\section{Results}

\section{Basic information}

There were 135 patients (1 male) with 210 SLNs detected in the SNV group and 68 patients with 102 SLNs detected in the SNZ group. Metastatic cases and metastatic LNs were identified as positive cases and positive LNs, respectively (Table 1).

\section{Success rate of SLN enhance}

Among 135 patients in SonoVue group, 1 case failed in CEUS localization and 4 cases failed in blue dye localization $(\mathrm{P}=0.370) ; 68$ patients in Sonazoid group, all succeeded in CEUS localization and 3 cases failed in blue dye $(\mathrm{P}=0.244)$. There was no significant difference between the successful rate of SonoVue group and Sonazoid group $(99.3 \% v s$. $100.0 \%, \mathrm{P}>0.05)$.

\section{Number of SLNs detected}

In the SNV group, CEUS failed to locate one SLN in one case. In the SNZ group, all SLNs were successfully located. Different numbers of SLNs were detected using the contrast agents and blue dye in the two groups. The SLN identification rates were $75.27 \%(210 / 279)$ for SNV and $93.58 \%(102 / 109)$ for SNZ. The median number of SNV-SLNs (SLNs identified using SNV) per patient was 1 (mean, 1.56; range, 1-4), and the median number of SNZSLNs (SLNs identified by SNZ) was 1 (mean, 1.50; range, 1-3) (Table 2). The numbers of SLNs detected using CEUS in the two groups and the corresponding number of SLNs were significantly lower than using blue dye $\left(t_{\mathrm{SNV}}=6.964\right.$, $\left.\mathrm{P}<0.001 ; t_{\mathrm{SNZ}}=3.763, \mathrm{P}<0.001\right)$.

Table 2 Comparison of SLNs detected by CEUS and blue dye in the two groups

\begin{tabular}{lcccccc}
\hline & \multicolumn{3}{c}{ SNV Group } & & \multicolumn{2}{c}{ SNZ Group } \\
\cline { 2 - 6 } \cline { 5 - 6 } & CEUS & Blue Dye & P & CEUS & Blue Dye & P \\
\hline Total number of SLNs & 210 & 297 & 0.000 & 102 & 139 \\
Mean number of SLNs & 1.56 & 2.20 & & 1.50 & 1.90 & \\
\hline
\end{tabular}

SNV, SonoVue ${ }^{\circledR}$; SNZ, Sonazoid ${ }^{\circledR}$; SLN: sentinel lymph node; CEUS, contrast-enhanced ultrasonography. 
Table 3 Sensitivity, specificity, PPV, and NPV of CEUS for the detection of metastasis in SLNs identified using SNV and SNZ

\begin{tabular}{lcc}
\hline $\begin{array}{l}\text { SLN Detection } \\
\text { Qualities }\end{array}$ & SNV & SNZ \\
\hline Sensitivity & $78.95 \%(30 / 38)$ & $88.46 \%(23 / 26)$ \\
Specificity & $94.77 \%(163 / 172)$ & $90.79 \%(69 / 76)$ \\
Accuracy & $91.90 \%(193 / 210)$ & $90.20 \%(92 / 102)$ \\
PPV & $76.92 \%(30 / 39)$ & $76.67 \%(23 / 30)$ \\
NPV & $95.31 \%(163 / 171)$ & $95.83 \%(69 / 72)$ \\
\hline
\end{tabular}

PPV, positive predictive value; NPV, negative predictive value; CEUS, contrast-enhanced ultrasonography; SLN, sentinel lymph node; SNV, SonoVue ${ }^{\circledR}$; SNZ, Sonazoid ${ }^{\circledR}$.

Table 4 Differences in metastasis of SLNs identified using SNV and SNZ

\begin{tabular}{lcccc}
\hline & True & False & P & F \\
\hline SNV & 193 & 17 & 0.615 & 0.254 \\
SNZ & 92 & 10 & & \\
\hline
\end{tabular}

True: diagnosis is consistent with the pathological findings; False: diagnosis is not consistent with the pathological findings. SLN: sentinel lymph node; SNV: SonoVue ${ }^{\circledR}$; SNZ: Sonazoid ${ }^{\circledR}$.

\section{Diagnosis of SLN metastasis}

Comparisons of metastatic SLNs detected with the two contrast agents are shown in Table 3. In group SonoVue, 210 out of 135 SLN patients were detected in 29 cases, 38 SLN diagnosed as SLN metastasis, 17 patients in 102 SLN detected by 69 patients in group Sonazoid, and 26 SLN diagnosed as SLN metastasis.

Of the 210 SNV-SLNs examined with CEUS, there were 30 true-positive and 163 true-negative results. The PPV, NPV, and accuracy were $76.92 \%, 95.31 \%$, and $91.90 \%$, respectively. In contrast, of the 102 SNV-SLNs examined, there were 23 true-positive and 69 true-negative results. The PPV, NPV, and accuracy were $76.67 \%, 95.83 \%$, and $90.20 \%$, respectively (Table 3). SLN metastasis was evaluated based on perfusion characteristics, and the accuracy of detecting SLN metastasis was slightly different between the two groups. The Pearson chi-square test showed no significant differences between the two groups $(\mathrm{P}=0.615)$ (Table 4).

\section{Enhancement duration and in vitro verification}

All excised SLNs were soaked in normal isotonic physiologic saline and compared with the LNs located by SNV/SNZ during the preoperative stage and with blue dye. All SNV-SLNs were not enhanced in isotonic saline, but all SNZ-SLNs were enhanced continuously and clearly until the end of operation (2-5 hours).

In SNZ group, the resected blue SLNs could be enhanced under CEUS condition, suggesting that these SLNs located by CEUS before operation were matched with blue SLNs (Figure 1). If blue-dye SLNs cannot be enhanced under CEUS, it indicates that SLNs is not completely resected and further exploration is needed to locate the SLNs. Only five cases underwent second intraoperative exploration and matched SLNs were found.

\section{Safety}

In this study, detection of SLNs using CEUS was performed under general anesthesia and immediately followed by SLNB and ALN dissection (ALND). Detection of SLNs using CEUS was helpful when performing surgery of the breast and minimized the patient's distress. No adverse reactions related to the periareolar injection of the two contrast agents, such as skin reactions around the injection site or allergic reactions, were observed immediately or at 1 month after surgery.

\section{Discussion}

ALND can be used to analyze the ALN status in patients with breast cancer. However, the meta-analysis results of van La Parra et al. (6) showed that ALND can only benefit $40-60 \%$ of SLN-positive patients. ALND destroys axillary lymph flow and may causes upper limb edema, numbness, shoulder joint dysfunction and pain (7). SLNB is widely recognized as an index for assessing axillary metastasis of tumors and a staging standard for clinical lymph node negative breast cancer patients. In previous studies, methylene blue localization is the most commonly used method to determine the location and number of axillary sentinel lymph nodes in breast surgery $(8,9)$. Previous studies have found that the combination of blue dye and isotope can make the rate of SLN localization to $96-99 \%$ (10). However, due to the increase of medical costs and the problems of radiation exposure and pollution, the clinical application of isotopes is limited, especially in developing countries. Blue dye also has some adverse reactions, including local skin reactions and allergic reactions, and cannot be applied to lactating and pregnant 

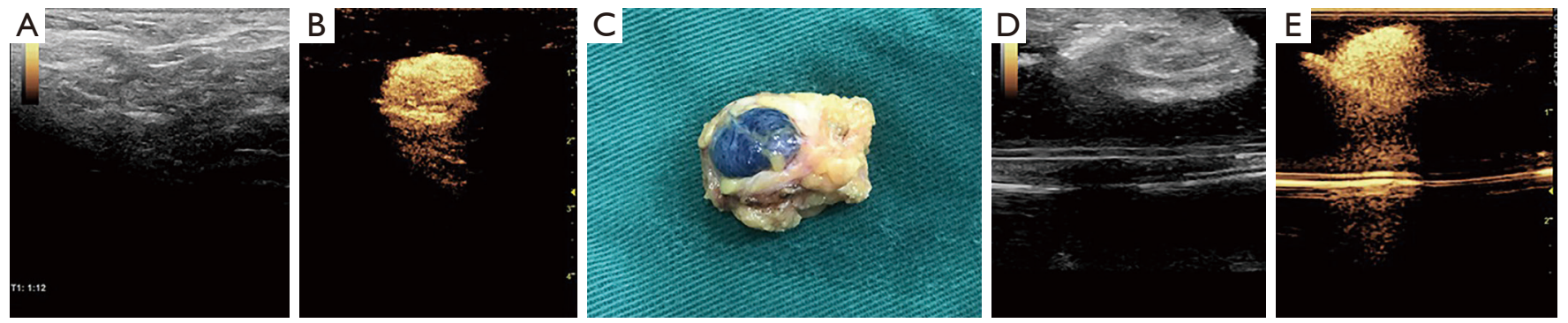

Figure 1 Invasive ductal carcinoma of the left breast of a 75-year-old woman (T1NOMO). Axillary lymph node detection using contrastenhanced ultrasonography. (A) and (B) are live dual images and macroscopic appearance of an SLN. (A) The SLN could not be observed on grayscale imaging. (B) The SLN were enhanced by CEUS. (C) show the resected blue SLN. (D) and (E) are live dual images of an sentinel lymphatic channel (SLC) and an sentinel lymphatic node (SLN)soaked in physiological saline.

women (11).

CEUS has undergone rapid development and can be used by surgeons as a road map for identifying SLN for SLNB purposes recently $(12,13)$. The size, number, location, distance to the body surface, and enhancement of the SLNs are documented, and the corresponding locations of the SLNs are marked on the body surface before SLNB. It has been proven that the efficacy of CEUS for SLN localization of early breast cancer before surgery is equivalent to that of blue dye during surgery (14). SNV was first used for the diagnosis of lesion in the liver, thyroid, kidney, and breast (15-20). SNV is a kind of microbubble contrast agent, which is encapsulated on lipid membrane by sulfur hexafluoride. SNZ is another microbubble contrast agent composed of phospholipid coated 10 fluorobutane. It was first used in clinical practice in Japan in 2007. In this study, we used it during the SLN localization study and compared the results with those of SNV. There was no significant difference in the SLN detection rates of breast cancer patients in the SNZ group and SNV group. Furthermore, there was no significant difference in the numbers of lymph nodes in the SNZ group and SNV group. Recent researches show that SNZ's shell is more rigid and stable than that of SNV (21) and can be used during high-frequency ultrasound examinations (22). Therefore, the application of $\mathrm{SNZ}$ in ultrasound examination of superficial tissue has a good prospect.

The mean numbers of SNV-SLNs (1.56) and SNZSLNs (1.50) were significantly lower than the mean number of SLNs identified using blue dye $(\mathrm{P}<0.05)$. Shimazu et al. (23) and Kenzo et al. (24) reported that the mean number of SNZ-SLNs was 1.52, which was also lower than the mean number of radiocolloid SLNs (2.19). The behavior of the tracer used in SLNB process is strongly dependent on its particle size. The median diameter of the SNV particles ranged from 1.5 to $2.5 \mu \mathrm{m}$, and that of SNZ particles ranged from 2.4 to $3.5 \mu \mathrm{m}$; both of these are larger than those of any other dye $(1.5-28 \mathrm{~nm})(25)$. Large particle tracer is not easy to enter the lymphatic system, but once trapped in lymph nodes, it will be retained for a long time, and the probability of entering non sentinel lymph nodes is very low (26). Therefore, the larger particle size of CEUS agents may partly explain the significantly lower mean number of SLNs obtained with the blue dye. Moreover, according to previous research habits, this study only recorded the first group of enhanced LNs as SLN, while the other ALN was not included in the number of SLN (27). The above two factors caused the SLN number of the CEUS agent group to be significantly lower than that of the blue dye group.

Metastatic LNs are characterized by internal peripheral and mixed microvessels due to tumor angiogenesis. However, color Doppler ultrasound can only provide information about the blood flow and morphology of large vessels, but cannot reflect the internal microvessels of tumor. Therefore, it is difficult to accurately diagnose LN metastasis using color Doppler ultrasound. Ultrasound contrast agent can show the micro blood flow well (28), so it can be used to evaluate the internal blood flow of LNs and provide more reliable image information for the diagnosis of metastatic LNs. SNV and SNZ are the second-generation contrast agents for US that allow visualization of LN microvessels. Liu et al. (29) reported that of 116 SLNs detected using percutaneous CEUS with SNV, the sensitivity and specificity for the diagnosis of metastatic SLN were $98.04 \%$ and $49.23 \%$, respectively. In another study that enrolled 101 cases and predicted SLN metastasis using CEUS enhancing patterns, the sensitivity 
was $81.8 \%$, specificity was $86.2 \%$, PPV was $75.0 \%$, and NPV was $90.3 \%$ (30). Shimazu et al. (23) reported that the accuracy of CEUS with SNZ for the diagnosis of metastatic SLN was $85.9 \%$ in a study with 98 patients. Matsuzawa et al. (31) analyzed the detection of metastasis based on contrast-enhanced computed tomography, color Doppler US, and CEUS with SNZ; their results suggested that CEUS with SNZ was the most accurate among these modalities.

Previous studies have shown the presence of defect areas and inhomogeneous or scarce perfusion in CEUS are seen as signs of neoplastic infiltration (32). Moreover, other literature has confirmed that tumor cells may infiltrate most lymph nodes, replace all normal tissues or block the main lymphatic vessels, leading to the failure of contrast medium. In these cases, sentinel lymph nodes showed weak or no enhancement (33). Accordingly, the LNs show uneven enhancement or incomplete ring enhancement, no enhancement, or weak enhancement of the node connected to the lymphatic vessel, they were considered metastatic LNs. The sensitivity, specificity, accuracy, PPV, and NPV for SLNs were evaluated based on the results of postoperative pathology. There was no significant difference in the evaluation of metastatic LNs of the SNV group and SNZ group.

SNZ and SNV are both based on the principle of harmonic signal of contrast agent microbubbles, but SNZ shell is a stable and negatively charged liposome component (34), which can simulate the distribution of liposomes on the surface of cell membrane and is easy to be engulfed by Kupffer cells in liver, while SNV has no such characteristics. Yanagisawa et al. (35) found that the phagocytic rate of SNZ on Kupffer cells was $99.00 \%$, while that of SNV on Kupffer cells was only 7.30\%. In addition, it has been confirmed that SNZ can be collected by the reticuloendothelial cells of LNs and detected in living tissues for a day (36,37). In this study, the SNZ group was able to continuous enhance and in vitro verification. Such outstanding stability is thought to allow longer lasting US examinations than those performed with other contrast agents (25). Therefore, the LNs resected during procedures can be used for imaging and comparative studies involving LNs located using SNZ and blue dye. If the SLN located with preoperative CEUS is consistent with the LN traced with blue dye during the procedure, then the biopsy is considered complete. If blue-stained LNs and LNs located using SNZ are not detected in saline, then the ALNs need to be explored further. The probe is aseptically treated to detect the LNs that developed near the operative incision and for preoperative localization of SLNs with continuous enhancement.

This study had some limitations. The number of cases evaluated was relatively small, and each group comprised a different number of patients. In addition, we cannot prove that only use of contrast agent is a safe way for SLNB, and we need a large sample and multi-center study. However, the results of this study may greatly increase the interest and participation of clinicians in exploring CEUS-SLN techniques, thereby providing the foundation for future large-scale clinical studies.

\section{Conclusions}

This study compared the subdermal uses of SNV and SNZ for SLN identification, and the results showed no significant differences in the number of SLNs detected or in the diagnosis of metastatic LNs. Furthermore, because SLNs can be detected for long periods after the injection of SNZ, it appears that SNZ-SLNs represent the true SLNs that are not affected by subjective factors related to the operators. Therefore, SNZ can be used to evaluate the circumstances of LN resection after surgery and provide an image-based location for timely feedback and repeat biopsy.

\section{Acknowledgments}

Funding: Key clinical projects in Peking University Third Hospital (No. Y78480-02).

\section{Footnote}

Reporting Checklist: The authors have completed the STARD reporting checklist. Available at http://dx.doi.org/10.21037/ gs-21-87

Data Sharing Statement: Available at http://dx.doi. org/10.21037/gs-21-87

Conflicts of Interest: All authors have completed the ICMJE uniform disclosure form (available at http://dx.doi. org/10.21037/gs-21-87). The authors have no conflicts of interest to declare.

Ethical Statement: The authors are accountable for all aspects of the work in ensuring that questions related to the accuracy or integrity of any part of the work are 
appropriately investigated and resolved. The study was conducted in accordance with the Declaration of Helsinki (as revised in 2013). All patients provided written informed consent for their information to be stored in the hospital database and used for research, and this study was approved by the Medical Ethics Committee of Peking University Third Hospital (item number M2017394).

Open Access Statement: This is an Open Access article distributed in accordance with the Creative Commons Attribution-NonCommercial-NoDerivs 4.0 International License (CC BY-NC-ND 4.0), which permits the noncommercial replication and distribution of the article with the strict proviso that no changes or edits are made and the original work is properly cited (including links to both the formal publication through the relevant DOI and the license). See: https://creativecommons.org/licenses/by-nc-nd/4.0/.

\section{References}

1. Kuwajerwala NK, Feczko C, Dekhne N, et al. Comparison of lymphedema in patients with axillary lymph node dissections to those with sentinel lymph node biopsy followed by immediate and delayed ALND. Am J Clin Oncol 2013;36:20-23.

2. Karakatsanis A, Daskalakis K, Stålberg P, et al. Superparamagnetic iron oxide nanoparticles as the sole method for sentinel node biopsy detection in patients with breast cancer. Br J Surg 2017;104:1675-85.

3. Dellaportas D, Koureas A, Contis J, et al. Contrast enhanced color doppler ultrasonography for preoperative evaluation of sentinel lymph node in breast cancer patients. Breast Care (Basel) 2015;10:331-5.

4. Lee JY, Minami Y, Choi BL, et al. The AFSUMB Consensus Statements and Recommendations for the Clinical Practice of Contrast-Enhanced Ultrasound using Sonazoid. Ultrasonography 2020;39:191-220.

5. Zhai HY, Liang P, Yu J, et al. Comparison of Sonazoid and SonoVue in the Diagnosis of Focal Liver Lesions: A Preliminary Study. J Ultrasound Med 2019;38:2417-25.

6. van la Parra RF, Peer PG, Ernst MF, et al. Meta-analysis of predictive factors for non-sentinel lymph node metastases in breast cancer patients with a positive SLN. Eur J Surg Oncol 2011;37:290-9.

7. Britton P, Willsher P, Taylor K, et al. Microbubble detection and ultrasound-guided vacuum-assisted biopsy of axillary lymph nodes in patients with breast cancer. Clin Radiol 2017;72:772-9.
8. Paulinelli RR, Freitas-Junior R, Raha RM, et al. A prospective randomized trial comparing patent blue and methylene blue for the detection of the sentinel lymph node in breast cancer patients. Rev Assoc Med Bras 2017;63:118-23.

9. Nandu VV, Chaudhari MS. Efficacy of Sentinel Lymph Node Biopsy in Detecting Axillary Metastasis in Breast Cancer Using Methylene Blue. Indian J Surg Oncol 2017;8:109-12.

10. Cui Q, Dai L, Li J, et al. Accuracy of CEUS-guided sentinel lymph node biopsy in early-stage breast cancer: a study review and meta-analysis. World J Surg Oncol 2020;18:112.

11. Somashekhar SP, Kumar CR, Ashwin KR, et al. Can Lowcost Indo Cyanine Green Florescence Technique for Sentinel Lymph Node Biopsy Replace Dual Dye (Radiocolloid and Blue Dye) Technique in Early Breast Cancer: A Prospective Two-arm Comparative Study. Clin Breast Cancer 2020;20:e576-e583.

12. Wang Y, Zhou WB, Li CY, et al. Variation of sentinel lymphatic channels (SLCs) and sentinel lymph nodes (SLNs) assessed by contrast-enhanced ultrasound (CEUS) in breast cancer patients. World J Surg Oncol 2017;15:127.

13. Haugen B R, Alexander E K, Bible K C, et al. 2015 American Thyroid Association Management Guidelines for Adult Patients with Thyroid Nodules and Differentiated Thyroid Cancer: The American Thyroid Association Guidelines Task Force on Thyroid Nodules and Differentiated Thyroid Cancer. Thyroid 2016;26:1-133.

14. Cox K, Taylor-Phillips S, Sharma N, et al. Enhanced pre-operative axillary staging using intradermal microbubbles and contrast-enhanced ultrasound to detect and biopsy sentinel lymph nodes in breast cancer: A potential replacement for axillary surgery. Br J Radiol 2018;91:20170626.

15. Zhang J, Zhang X, Meng Y, et al. Contrast-enhanced ultrasound for the differential diagnosis of thyroid nodules: An updated meta-analysis with comprehensive heterogeneity analysis. PLoS One 2020;15:e0231775.

16. Tang C, Fang K, Guo Y, et al. Safety of sulfur hexafluoride microbubbles in sonography of abdominal and superficial organs: Retrospective analysis of 30,222 cases. J Ultrasound Med 2017;36:531-8.

17. Tenant SC, Gutteridge CM. The clinical use of contrast-enhanced ultrasound in the kidney. Ultrasound 2016;24:94-103.

18. Ma JJ, Ding H, Xu BH, et al. Diagnostic performances of various gray-scale, color Doppler, and contrast-enhanced 
ultrasonography findings in predicting malignant thyroid nodules. Thyroid 2014;24:355-63.

19. Li CY, Gong HY, Ling LJ, et al. Diagnostic performance of contrast-enhanced ultrasound and enhanced magnetic resonance for breast nodules. J Biomed Res 2018;32:198-207.

20. Barr RG, Huang PT, Yan Luo, et al. Contrast-enhanced ultrasound imaging of the liver: a review of the clinical evidence for SonoVue and Sonazoid. Abdom Radiol (NY) 2020;45:3779-88.

21. Jiang Y, Lv K, Liang P, et al. A Phase 3 Multicentre, Randomised, Comparative Study of the Efficacy and Safety of Sonazoid and SonoVue in Subjects with Focal Liver Lesions Undergoing Pre- and Post-Contrast Ultrasound Imaging. Ultrasound Med Biol 2017;43:S34-S35.

22. Chou YH, Liang JD. Wang SY, et al. Safety of perfuorobutane (Sonazoid) in characterizing focal liver lesions. J Med Ultrasound 2019;27:81-5.

23. Shimazu K, Ito T, Uji K, Miyake T, et al. Identification of sentinel lymph nodes by contrast-enhanced ultrasonography with Sonazoid in patients with breast cancer: a feasibility study in three hospitals. Cancer Med 2017;6:1915-22.

24. Shimazu K, Miyake T, Tanei T, et al. Real-time visualization of lymphatic flow to sentinel lymph nodes by contrast-enhanced ultrasonography with Sonazoid in patients with breast cancer. Ultrasound Med Biol 2019;45:2634-40.

25. Sontum PC. Physicochemical characteristics of Sonazoid, a new contrast agent for ultrasound imaging. Ultrasound Med Biol 2008;34:824-33.

26. Kawai Y, Ajima K, Nagai T, et al. Real-time imaging of the lymphatic channels and sentinel lymph nodes of the stomach using contrast-enhanced ultrasonography with Sonazoid in a porcine model. Cancer Sci 2011;102:2073-81.

27. Li H, Jun Z, Cheng GZ, et al. Factors that affect the false negative rate of sentinel lymph node mapping with methylene blue dye alone in breast cancer. J Int Med Res 2019;47:4841-53.

28. Trimboli P, Castellana M, Virili C, et al. Performance

Cite this article as: Sun Y, Cui L, Wang S, Shi T, Hao Y, Lei Y. Comparative study of two contrast agents for intraoperative identification of sentinel lymph nodes in patients with early breast cancer. Gland Surg 2021;10(5):1638-1645. doi: 10.21037/ gs-21-87 of contrast-enhanced ultrasound (CEUS) in assessing thyroid nodules: a systematic review and meta-analysis using histological standard of reference. Radiol Med 2020;125:406-15.

29. Liu J, Liu X, He J, et al. Percutaneous contrast-enhanced ultrasound for localization and diagnosis of sentinel lymph node in early breast cancer. Sci Rep 2019;9:13545 .

30. Xie F, Zhang DJ, Cheng L, et al. Intradermal microbubbles and contrast-enhanced ultrasound (CEUS) is a feasible approach for sentinel lymph node identification in earlystage breast cancer. World J Surg Oncol 2015;13:319.

31. Matsuzawa F, Omoto K, Einama T, et al. Accurate evaluation of axillary sentinel lymph node metastasis using contrast-enhanced ultrasonography with Sonazoid in breast cancer: a preliminary clinical trial. Springerplus 2015;4:509.

32. Rubaltelli L, Khadivi Y, Tregnaghi A, et al. Evaluation of lymph node perfusion using continuous mode harmonic ultrasoography with a second-generation contrast agent. J Ultrasound Med 2004;23:829-36.

33. Aoki T, Moriyasu F, Yamamoto K, et al. Image of tumor metastasis and inflammatory lymph node enlargement by contrast-enhanced ultrasonography. World J Radiol 2011;3:298-305.

34. Maruyama T, Sugii M, Omata D, et al. Effect of lipid shell composition in DSPG-based microbubbles on blood flow imaging with ultrasonography. Int J Pharm 2020;590:119886.

35. Yanagisawa K, Moriyasu F, Miyahara T, et al. Phagocytosis of ultrasound contrast agent microbubbles by Kupffer cells. Ultrasound Med Biol 2007;33:318.

36. Watanabe R, Matsumura M, Munemasa T, et al. Mechanism of hepatic parenchyma-specific contrast of microbubble-based contrast agent for ultrasonography: microscopic studies in rat liver. Invest Radiol 2007;42:643-51.

37. Machado P, Stanczak M, Liu JB, et al. Subdermal ultrasound contrast agent injection for sentinel lymph node identification: an analysis of safety and contrast agent dose in healthy volunteers. J Ultrasound Med 2018;37:1611-20. 